\title{
CONSTRUCTAL DESIGN APPLIED TO THE OPTIMIZATION OF HEAT TRANSFER IN A SOLID CONDUCTING WALL
}

\author{
Marques, C. H. ${ }^{1}$, Dos Santos, E.D. ${ }^{2}$, Rocha, L.A.O. ${ }^{1{ }^{*}}$ \\ ${ }^{1}$ Program of Post-graduation in Computational Modeling, Universidade Federal do \\ Rio Grande, Italia Avenue, km 8, Cx.P. 474, Rio Grande, RS, Brasil, 96201-900. \\ ${ }^{2}$ Department of Mechanical Engineering, Universidade Federal do Rio Grande do \\ Sul, Sarmento Leite Street, 425, Porto Alegre, RS, Brasil, 90050-170.
}

\begin{abstract}
The present paper applies Constructal Design to optimize the geometry of a Yshaped negative fin that intrudes a solid conducting wall with heat generation. The main goal is the minimization of the thermal global resistance between the solid wall and the negative fin, which removes energy from the wall. The optimization is achieved by varying the angle between the tributary branch of the Y-Shaped fin and the horizontal axis, as well as, by varying the ratio between the volume of the fin and the rectangular volume that circumscribes it $(\psi)$, while the other geometric parameters are maintained fixed. Constructal Design led to a best configuration, with a thermal global resistance of $53 \%, 49 \%$ and $48 \%$ for $\psi=0.3, \psi=0.4$ and $\psi=0.5$, respectively, smaller than the ones for the worst configuration.
\end{abstract}

Keywords: Constructal Design; Thermal optimization; Y-Shaped; Negative Fin.

"Corresponding author: laorocha@gmail.com Phone: +5553 32336883 


\section{INTRODUCTION}

Constructal Design has been applied to a large variety of engineering problems, e.g., (Bejan, 1997; Vargas et al., 2005; Bejan and Dan, 1999; Rocha et al., 2003) in order to optimize shape and structure (Bejan, 2000). Concerning the study of fins, the optimization of T-shaped assembly of fins was performed in Bejan and Almogbel (2000) and Y-shaped fins in Lorenzini and Rocha (2006). On the other hand, cavities, also known as inverted or negative fins, were studied by Biserni et al. (2004) for the optimization of C- and T-shaped ones. For both situations it was minimized the global thermal resistance while the total volume and the cavity volume were kept as constrains. However, studies about Y-shaped cavities inserted into a solid conducting wall with heat generation have not been presented at the present moment, by the authors knowledge, being the scope of the present work.

This kind of geometry is important in practically every domain where augmentation and compactness (high density) of heat transfer are required. Additionally, according to Biserni et al. (2004), open cavities are the regions formed between adjacent fins. Then, if the optimization of the geometry between individual fins is an important issue, certainly, the geometry of the interstices must also be important. Open cavities are essential promoters of nucleate boiling and condensation. In nature, open cavities could be used in order to represent the alveolus of the lung, where the relatively robust (not slender) shape of the cavity has been noted and attributed intuitively to the natural Constructal optimization principle.

The present work applies Constructal Design to optimize the heat exchange between a conducting solid wall with heat generation and a Y-shaped inverted fin to remove energy from the former. The angle between a tributary branch and the horizontal axis varies for various values of the ratio between the volume of the $\mathrm{Y}$ shaped cavity and the rectangular volume that circumscribes it $(\psi=0.3,0.4$ and 0.5$)$ being the degrees of freedom of the geometry. The objective is to minimize the global thermal resistance in the solid wall subjected to a fixed ratio between the volume of Y-shaped inversed fin and the volume of the total solid.

\section{MATHEMATICAL MODEL}

The analysed physical problem consists of a conducting solid wall with a $\mathrm{Y}$ shaped inverted fin intruded in the solid domain, as can be seen in FIGURE 1. The solid is isotropic with a constant thermal conductivity $k(\mathrm{~W} / \mathrm{mK})$. It generates heat 
uniformly at the volumetric rate $q^{\prime \prime \prime}\left(\mathrm{W} / \mathrm{m}^{3}\right)$. The outer surfaces of the heat generating body are perfectly insulated. The generated heat current is removed by cooling the wall with a $\mathrm{Y}$-shaped negative fin. This one is maintained at $T_{\min }$ and the temperatures in the solid are higher than $T_{\text {min }}$. The highest temperatures (the "hot spots") are registered at points on the adiabatic perimeter, for instance, in the corners of solid wall. Two elemental cavities of thickness $t_{0}$ and length $L_{0}$ serve as tributaries to a stem of thickness $t_{1}$ and length $L_{1}$. The elemental cavity fin of thickness $t_{0}$ forms an angle $\alpha$ with a horizontal line. Besides that, the problem is considered twodimensional, with the third dimension $(W$ ) sufficiently long in comparison with the other dimensions of the solid domain and the inverted fin.

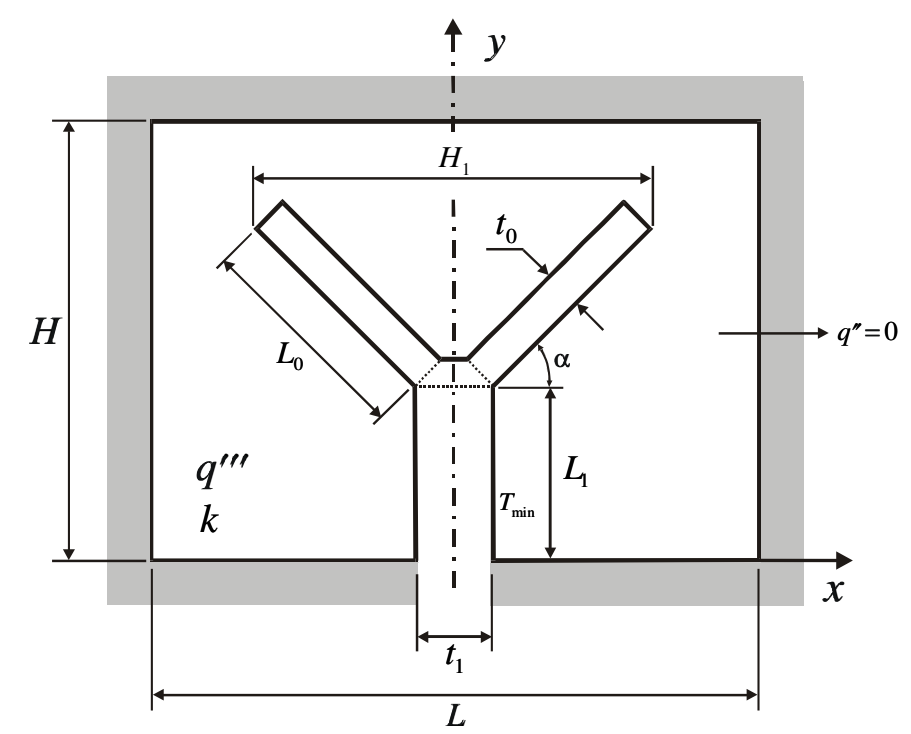

Figure 1 - Physical domain of Y-shaped inverted fin inside rectangular volume with heat generation.

The objective of the present analysis is to determine the optimal geometry that is characterized by the minimum global thermal resistance $\left(T_{\max }-T_{\min }\right) /\left(q^{\prime \prime \prime} A\right)$. To achieve this goal, the angle $\alpha$ varies and the system is subjected to three constraints. The first constraint is the volume of the solid wall (i.e., frontal area), given by:

$$
A=H L
$$

The second one is the volume of $\mathrm{Y}$-shaped cavity, which is given by:

$$
A_{f}=L_{1} t_{1}+2 L_{0} t_{0}+t_{1} t_{0} \cos \alpha-t_{0}^{2} \sin \alpha \cos \alpha
$$

Eq. (2) can be expressed as the fin volume fraction, which can be written by: 


$$
\phi=\frac{A_{t}}{A}
$$

The last constraint is the volume that circumscribes the inverted fin, which is given by:

$$
A_{1}=\left(L_{1}+L_{0} \sin \alpha+t_{0} \cos \alpha\right)\left(2 L_{0} \cos \alpha+t_{1}\right)
$$

The latter constraint is related with the volume of the $\mathrm{Y}$-shaped cavity by the following expression:

$$
\psi=\frac{A_{t}}{A_{1}}
$$

The numerical optimization of geometry consisted of simulating the temperature field in a large number of configurations, calculating the global thermal resistance for each configuration, and selecting the configuration with the smallest global resistance. The dimensionless conduction equation solved in the solid wall is given by:

$$
\frac{\partial^{2} \theta}{\partial \tilde{x}^{2}}+\frac{\partial^{2} \theta}{\partial \tilde{y}^{2}}+1=0
$$

where the dimensionless variables are:

$$
\begin{aligned}
& \theta=\frac{T-T_{\text {min }}}{q^{\prime \prime \prime} A / k} \\
& \tilde{H}, \tilde{L}, \tilde{t}_{0}, \tilde{L}_{0}, \tilde{t}_{1}, \widetilde{L}_{1}=\frac{H, L, t_{0}, L_{0}, t_{1}, L_{L}}{A^{1 / 2}}
\end{aligned}
$$

The constraints, given by Eq. (1), (3) and (5) are also written, respectively, in its dimensionless form by the following expressions:

$$
\begin{aligned}
& \tilde{H} \tilde{L}=1 \\
& \phi=\tilde{L}_{1} \tilde{t}_{1}+2 \tilde{L}_{0} \tilde{t}_{0}+\tilde{t}_{1} \tilde{t}_{0} \cos \alpha-\tilde{t}_{0}^{2} \sin \alpha \cos \alpha \\
& \psi=\left(\tilde{L}_{1}+\tilde{L}_{0} \sin \alpha+\tilde{t}_{0} \cos \alpha\right)\left(2 \tilde{L}_{0} \cos \alpha+\tilde{t}_{1}\right)
\end{aligned}
$$

\section{NUMERICAL MODEL}

The function defined by Eq. (7) can be numerically determined by the solution of Eq. (6) for the temperature field as function of every degree of freedom $\left(L_{1} / L_{0}, t_{1} / t_{0}\right.$, $\alpha, H / L)$ subjected to the constraints $(A, \phi, \psi)$, and calculating $T_{\max }$ to see whether this variable can be minimized by varying the configuration. In order to obtain the numerical solution, Eq. (4) was solved using a finite element code, based on triangular elements, developed in MATLAB environment, precisely the PDE (partial- 
differential-equations) toolbox (MATLAB, 2000). This numerical code was previously validated by Lorenzini and Rocha (2006) and, for the sake of simplicity, it will not be evaluated again in the present work. The grid was non-uniform in both $\tilde{x}$ and $\tilde{y}$ directions, and varied from one geometry to the next. The appropriate mesh size was determined by successive refinements until the criterion, $\left|\left(\theta_{j \max }-\theta_{j+1 \max }\right) / \theta_{j \max }\right|<$ $5.0 \times 10^{-4}$, for the grid independence, has been satisfied. In the present work, the superscript $j$ represents the maximum temperature estimated using the current mesh size, and $j+1$ refers to the calculation for the next mesh size. It is also observed that the number of elements from the current mesh to the next mesh increases by four times. Table 1 illustrates how grid independence test was performed for the following parameters $\left(H / L=1 ; t_{1} / t_{0}=2, L_{1} / L_{0}=0.5, \phi=0.05, \psi=0.5\right.$ and $\left.\alpha=0.94\right)$. The results employed to optimization of geometry were obtained using a 35840 triangular elements mesh.

Table 1 - Numerical tests showing the achievement of grid independent for the following parameters $\left(H / L=1 ; t_{1} / t_{0}=2, L_{1} / L_{0}=0.5, \phi=0.05, \psi=0.5\right.$ and $\left.\alpha=0.94\right)-$ criterion $=5.0 \times 10^{-4}$.

\begin{tabular}{ccc}
\hline Number of Elements & $\boldsymbol{\theta}_{\max }$ & $\left|\left(\theta_{\max }^{\mathrm{j}}-\boldsymbol{\theta}^{\mathrm{j}+1}{ }_{\max }\right) / \boldsymbol{\theta}_{\max }^{\mathrm{j}}\right|$ \\
\hline 560 & 0.074760 & $2.24 \mathrm{E}-03$ \\
\hline 2240 & 0.074539 & $6.85 \mathrm{E}-04$ \\
\hline 8960 & 0.074487 & $1.73 \mathrm{E}-04$ \\
\hline 35840 & 0.074475 & $3.74 \mathrm{E}-05$ \\
\hline 143360 & 0.074472 & ------------ \\
\hline
\end{tabular}

\section{RESULTS}

The temperature fields were obtained for a large number of configurations, in order to investigate the effect of the angle between the tributary branches in the global thermal resistance of the conducting solid wall. In order to perform this investigation, the other parameters were maintained fixed: $H / L=1 ; t_{1} / t_{0}=2, L_{1} / L_{0}=$ $0.5, \phi=0.05, \psi=0.5$.

The temperature topology for the first case evaluated was presented in FIGURE 2. FIGURE 2A presents the configuration for an angle $\alpha=0.5 \mathrm{rad}$, which represents the minimal angle of the tributary branch with the horizontal axis $x$. 
FIGURE 2B shows the temperature topology for an intermediate angle of $\alpha=0.938$ $\mathrm{rad}$, and FIGURE $2 \mathrm{C}$ presents the configuration for an angle $\alpha=1.2 \mathrm{rad}$, which represents the other extreme situation, where the tributary branch has the highest angle with the horizontal axis. For the lower angle, FIGURE 2A, it is observed that the heat transfer occurs in a non-uniform way, in other words, the transfer occurs from the lower to the higher conductivity material mainly in the lower portion of the conducting solid wall. Besides that, it is observed only one hot spot in the upper part of solid wall. In FIGURE 2B, the heat transfer happens more equally along the solid domain, being observed three points where the global thermal resistance is the maximum.

However, in these three points the resistance is significantly lower than the one point of maximum for the former angle. In FIGURE 2.3B, it is observed that after one specific point for the angle the topology of temperature field becomes nonuniformly again, increasing the maximum thermal resistance. Therefore, there is one specific angle that optimizes the transfer of energy from the conducting solid wall to the cooled fin. Moreover, the best angle, in the present case $\alpha_{\mathrm{opt}}=0.938 \mathrm{rad}$, is the one that distributes uniformly the hot spots, i.e., the imperfections along the conducting solid wall. This conclusion is in agreement with the observations performed previously in Bejan (2000).

FIGURE $3 \mathrm{~A}, 3 \mathrm{~B}$ and $3 \mathrm{C}$ show the optimal temperature topologies for $\psi=0.3$, 0.4 and 0.5 , respectively. Evidently, when $\psi=0.5$ the minimal thermal global resistance is achieved. It is also observed that the optimal angle is inversely proportional to $\psi$. In fact, the system naturally redistributes its geometrical configuration with the purpose to reach a more uniformly distribution of the thermal field. An analogous observation was performed by Lorenzini and Rocha (2006) for the study of $Y$-shaped fins.

In order to evaluate quantitatively the performance of the cooling system as function of the angle between the tributary angle and the horizontal axis, it is obtained the maximum global thermal resistance for each simulated case. FIGURE 4 presents the optimization of the case with the following parameters $\left(H / L=1 ; t_{1} / t_{0}=2\right.$, $L_{1} / L_{0}=0.5, \phi=0.05, \psi=0.5$ ). The results corroborate the statements observed in the temperatures topologies, i.e., there is one angle that led to the best performance of the system. Besides that, the global thermal resistance for the best situation 
(optimal) is approximately $48 \%$ lower than the one for the worst situation, showing the importance of the employment of Constructal Design in engineering systems.

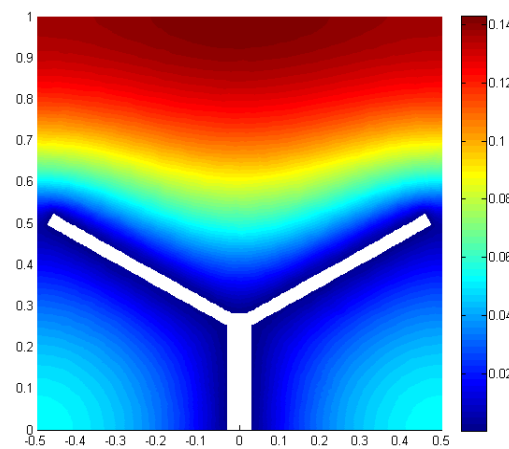

a)

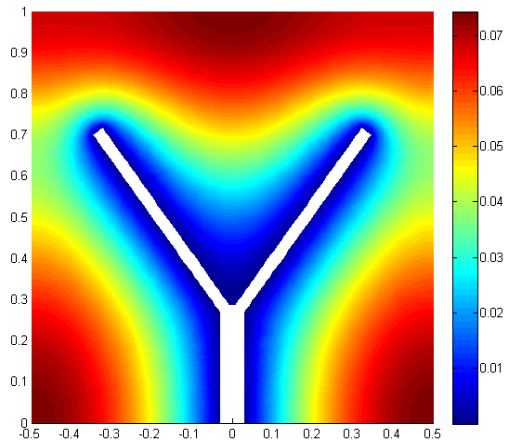

Optimal Geometry

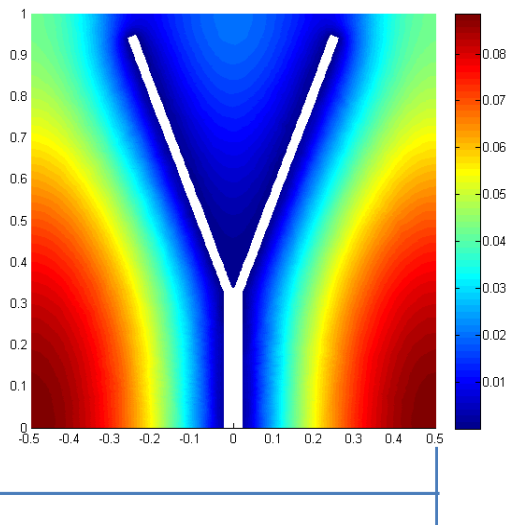

c)

Figure 2 - Temperature distribution in the cavity as function of angle $\alpha$ for $H / L=1$; $t_{1} / t_{0}=2, L_{1} / L_{0}=0.5, \phi=0.05, \psi=0.5$ : (a) $\alpha=0.5 \mathrm{rad}$, (b) $\alpha_{\mathrm{opt}}=0.938 \mathrm{rad}$, (c) $\alpha=1.2$ rad.

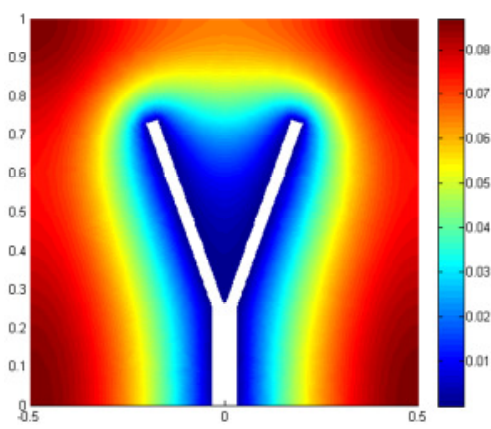

a)

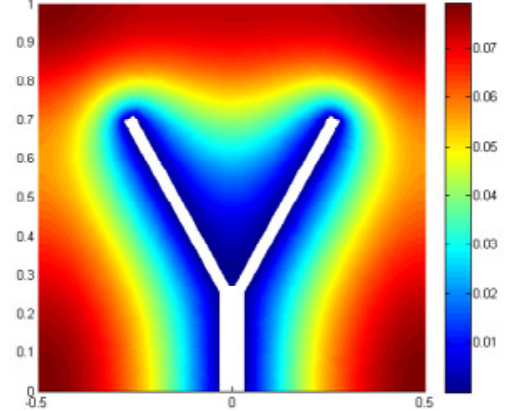

b)

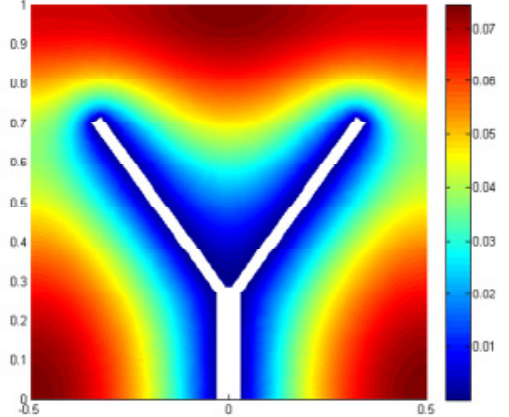

c)

Figure 3 - Optimal temperature distribution in the cavity as function of $\psi$ for $H / L=1$;

$$
t_{1} / t_{0}=2, L_{1} / L_{0}=0.5, \phi=0.05 \text { : (a) } \psi=0.3 \text {, (b) } \psi=0.4 \text {, (c) } \psi=0.5 \text {. }
$$

FIGURE 5 shows the minimization of the global thermal resistance as function of the angle between the tributary branch of the Y-shaped cavity and the horizontal axis $(\alpha)$ for various values of $\psi=0.3,0.4$ and 0.5 . Constructal Design led to a best configuration, with a thermal global resistance of $53 \%, 49 \%$ and $48 \%$ for $\psi=0.3, \psi=$ 0.4 and $\psi=0.5$, respectively, smaller than the ones for the worst configuration.

FIGURE 5 also indicates that, in general, for higher values of $\psi$ there are lower values of $\theta_{\max }$ if the angle $\alpha$ is kept constant. Nevertheless, it is not a true at all, for instance, for the range $1.05 \leq \alpha \leq 1.2$ the global thermal resistance obtained for $\psi$ 
$=0.4$ are lower than those predicted for $\psi=0.5$. This points the importance of apply Constructal Design, since it is possible to absorb more heat with a more compacted system. Spite of this fact, the minimal global thermal resistance is lower for higher values of $\psi$. For $\psi=0.5$ the minimal global thermal resistance was approximately $6 \%$ lower than for $\psi=0.4$, which subsequently was around $9 \%$ lower than $\psi=0.3$. Besides that, it is possible to perform a comparison between a T-shaped inverted fin intruded in a conduction solid wall, a Y-shaped inverted fin and a single slab (without a first construction). Once, the optimal angle was not the lowest nor the highest angle, which represent a $\mathrm{T}$-shaped negative fin and $\mathrm{C}$-shaped cavity, respectively. Therefore, it is possible to state that the Y-shaped inverted fin led to better performance than the other geometries compared.

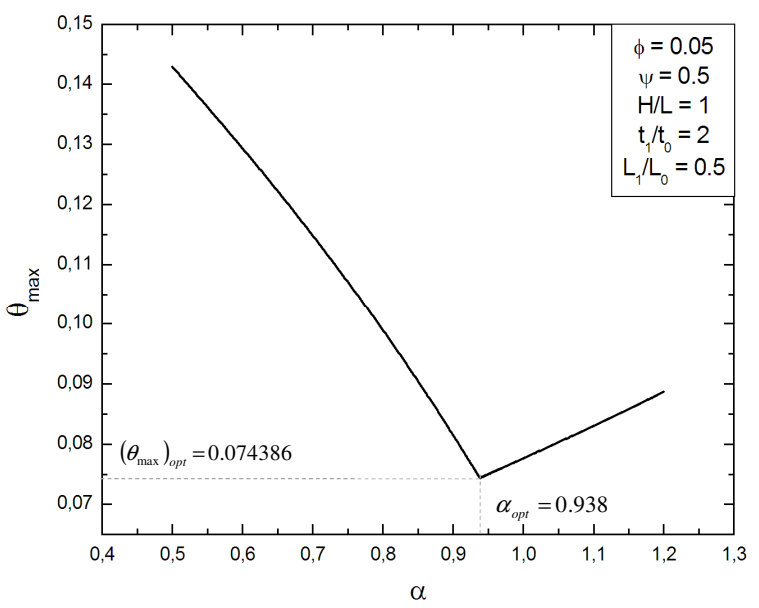

Figure 4 - The minimization of the global thermal resistance as function of angle $\alpha$ $\left(H / L=1 ; t_{1} / t_{0}=2, L_{1} / L_{0}=0.5, \phi=0.05, \psi=0.5\right)$.

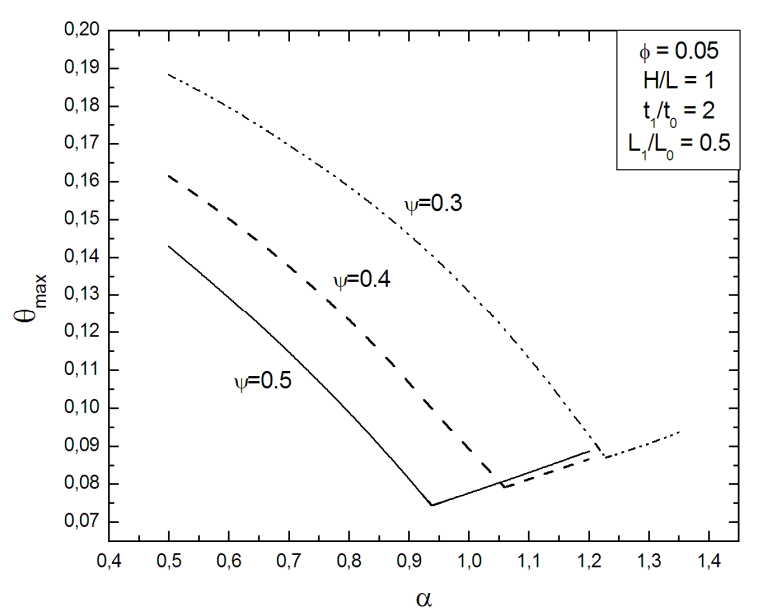

Figure 5 - The minimization of thermal resistance as function of angle $\alpha\left(H / L=1 ; t_{1} / t_{0}\right.$ $\left.=2, L_{1} / L_{0}=0.5, \phi=0.05\right)$ for: $\psi=0.3,0.4$ and 0.5 . 
FIGURE 6 presents the optimal global thermal resistance as function of $\psi$. This graph reinforces the findings of the previous figure. An comparison between the best and the worst situation point, $\psi=0.5$ and $\psi=0.3$, respectively, indicates a diference of around $15 \%$. The optimal global thermal resistance for $\psi=0.3$ was $\left(\theta_{\max }\right)_{\mathrm{opt}}=0.086992$, for $\psi=0.4$ was $\left(\theta_{\max }\right)_{\mathrm{opt}}=0.079157$ and for $\psi=0.5$ was $\left(\theta_{\max }\right)_{\mathrm{opt}}$ $=0.074386$.

FIGURE 7 ilustrates the optimal angle $\alpha_{\text {opt }}$ as function of $\psi$. Can be observed an steep decrease of the optimal angle $\alpha$ with the increase of the $\psi$. For $\psi=0.3$ the optimal value of the angle was $\alpha_{\mathrm{opt}}=1.228 \mathrm{rad}$, for $\psi=0.4$ the optimal angle was $\alpha_{\mathrm{opt}}$ $=1.060 \mathrm{rad}$ and for $\psi=0.4$ the optimal angle was $\alpha_{\mathrm{opt}}=0.938$.

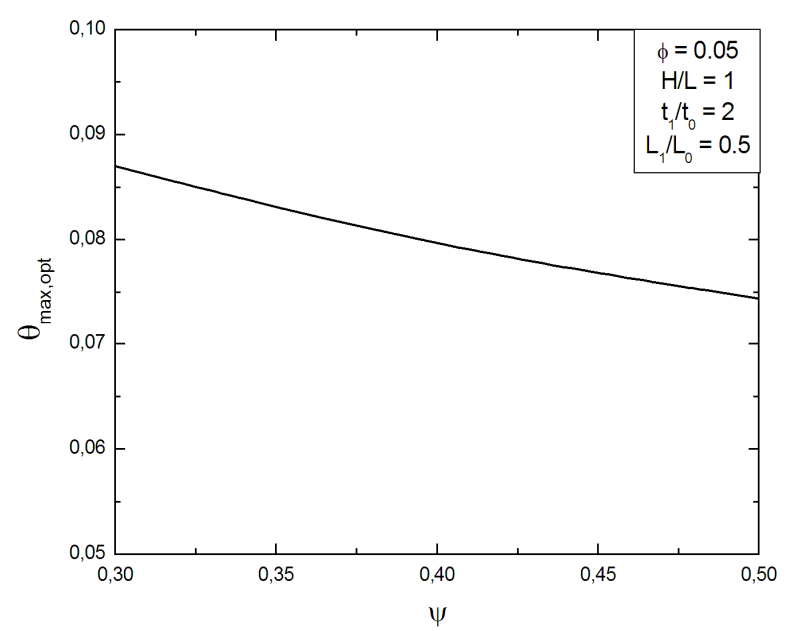

Figure 6 - The optimal global thermal resistance as function of $\psi$ for the following parameters $\left(H / L=1 ; t_{1} / t_{0}=2, L_{1} / L_{0}=0.5, \phi=0.05\right)$.

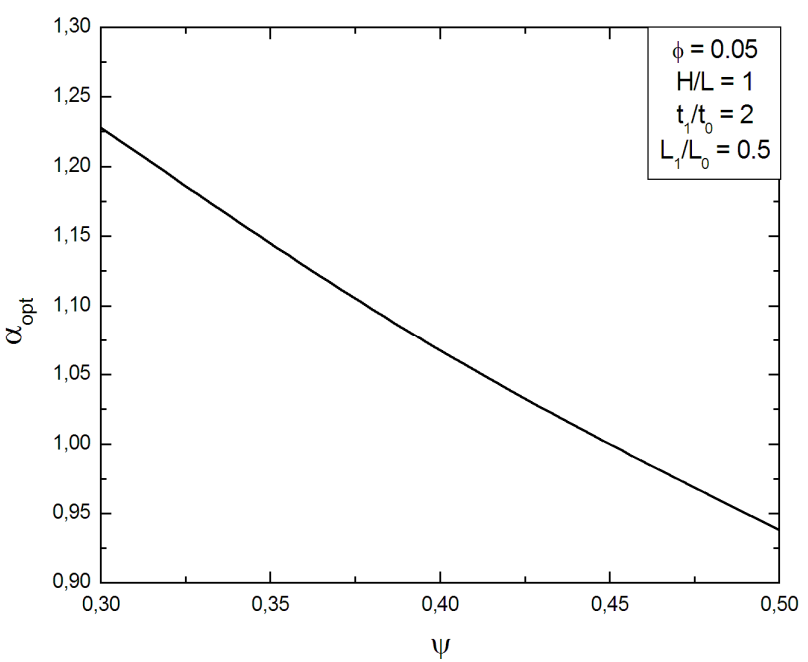

Figure 7 - The optimal angle $\alpha$ as function of $\psi$ for the following parameters $(H / L=1$;

$$
\left.t_{1} / t_{0}=2, L_{1} / L_{0}=0.5, \phi=0.05\right) \text {. }
$$




\section{CONCLUSIONS}

In the present work was performed a numerical study about the optimization of the geometry of a Y-shaped negative fin inserted in a solid conducting wall with heat generation by means of Constructal Design. The optimization was performed by varying the angle between the tributary branch of the $\mathrm{Y}$-shaped inverted fin and the horizontal axis for several ratios between the volume of the cavity and the rectangular volume that circumscribes it, $\psi=0.3, \psi=0.4$ and $\psi=0.5$.

For the same values of $\psi$, it was observed that there was one specific angle $\alpha$ that optimized the transfer of energy from the conducting solid wall to the cooled cavity. It was also noted that the optimal angle is the one that distributes more uniformly the hot spots along the conducting solid wall. This observation was in agreement with the principle of distribution of imperfections stated by Bejan (2000).

Constructal Design led to a best configuration, with a thermal global resistance of $53 \%, 49 \%$ and $48 \%$ for $\psi=0.3, \psi=0.4$ and $\psi=0.5$, respectively, smaller than the ones for the worst configuration.

For higher values of $\psi$ there are lower values of the global thermal resistance $\theta_{\max }$. However, for the range $1.05 \leq \alpha \leq 1.2$ the global thermal resistance obtained for $\psi=0.4$ are lower than those predicted for $\psi=0.5$, showing the employment of Constructal Design is important in order to avoid wastes, since it is possible to absorb more heat with more compacted systems.

In comparison with a T-shaped and C-shaped cavities, it is possible to state that the $\mathrm{Y}$-shaped inverted fin led to better performance than the other ones.

The optimal angle aopt deply decreases with the increase of $\psi$, showing that the system naturally redistributes its geometrical configuration to reach a more uniformly distribution of the thermal field, which is in agreement with the observations of Lorenzini and Rocha (2006) in the study of Y-shaped fins.

\section{REFERENCES}

BEJAN, A, ALMOGBEL, M., Constructal T-shaped fins, Int. J. Heat Mass Transfer, Vol. 43, 2000, pp. 2101-2115.

BEJAN, A., Constructal-Theory network of conducting paths for cooling a heat generating volume, Int. J. Heat Mass Transfer, Vol. 40, 1997, pp. 799 - 816. 
BEJAN, A., DAN, N., Two constructal routes to minimal heat flow resistance via greater internal complexity, J. Heat Transfer, Vol. 121, 1999, pp. 6-14.

BEJAN, A., Shape and Structure, from Engineering to Nature, Cambridge University Press, UK, 2000.

BISERNI, C., ROCHA, L.A.O., BEJAN, A., Inverted fins: geometric optimization of the intrusion into a conducting wall, Int. J. Heat Mass Transfer, Vol. 47, 2004, pp. 2577-2586.

LORENZINI, G., ROCHA, L.A.O., Constructal design of Y-shaped assembly of fins, Int. J. Heat Mass Transfer, Vol. 49, 2006, pp. 4552-4557.

MATLAB, User's guide, version 6.0.088, release 12, The Mathworks Inc., 2000.

ROCHA, L.A.O., LORENTE, S., BEJAN, A., Constructal design for cooling a discshaped are by conduction, Int. J. Heat Mass Transfer, Vol. 45 (8), 2003, pp. $1642-1652$.

VARGAS, J.V.C., ORDOÑEZ, J.C., BEJAN, A., Constructal PEM fuel cell stack design, Int. J. Heat Mass Transfer, Vol. 48, 2005, pp. 4410 - 4427.

\section{ACKNOWLEDGEMENTS}

E. D. dos Santos thanks CAPES by his doctorate scholarship and L. A. O. Rocha thanks CNPQ for his research grant. 